\title{
Die neue anatomische Flügelplatte für osteoporotische Azetabulumfrakturen: biomechanische Testung und erste klinische Erfahrungen
}

\author{
A New Anatomical Wing Plate for Osteoporotic Acetabular Fractures: \\ Biomechanical Testing and First Clinical Experience
}

Autoren

Institut
A. Schäffler, S. Döbele, F. Stuby, M. Maucher, S. Schröter, U. Stöckle, B. König

Klinik für Unfall- und Wiederherstellungschirurgie der Eberhard-Karls-Universität Tübingen, BG Unfallklinik Tübingen

\author{
Schlüsselwörter \\ - Azetabulumfraktur \\ - Flügelplatte \\ optisches Messsystem \\ - Stoppa-Zugang \\ - Beckeneinbeinstand \\ Key words \\ - acetabular fracture \\ - wing plate \\ - optoelectronic 3D camera \\ measuring system \\ - Stoppa approach \\ - one-leg stand pelvic model
}

Bibliografie

DOI http://dx.doi.org/

10.1055/s-0033-1360351

Z Orthop Unfall 2014; 152:

26-32 @ Georg Thieme

Verlag KG Stuttgart · New York .

ISSN 1864-6697

Korrespondenzadresse Dr. Aljoscha Schäffler Klinik für Unfall- und Wiederherstellungschirurgie der Eberhard-Karls-Universität Tübingen BG Unfallklinik Tübingen Schnarrenbergstraße 95 72076 Tübingen

Tel.: 07071/6063022

aschaeffler@bgu-tuebingen.de

\section{License terms}

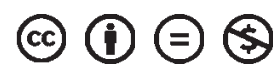

\section{Zusammenfassung}

$\nabla$

Einleitung: Generell stellen Frakturen des Azetabulums eine seltene Verletzung dar. Deutlich zunehmend ist aber die Zahl der Azetabulumfrakturen im Alter mit vornehmlich ventraler Pathologie und medialer Protrusion des Hüftkopfs. Die herkömmliche Plattenosteosynthese kann hier medialseitig keine flächige Abstützung bieten. Die von uns entwickelte Platte soll anatomisch vorkonturiert die Reposition erleichtern, eine flächige Abstützung der quadrilateralen Fläche ermöglichen und so der nach innen drängenden Kraft des Hüftkopfs entgegenwirken. Ziel dieser Studie war die biomechanische Testung der neuen Platte vor der klinischen Einführung.

Material und Methoden: Für die biomechanische Testung wurden 8 Frakturmodelle des Beckens der Firma Synbone ${ }^{\circledR}$ (vorderer Pfeiler) verwendet. Es wurde der Einbeinstand simuliert und in einer Materialprüfmaschine eine zyklische Messung mit schrittweiser Drucksteigerung bis zu $250 \mathrm{~N}$ durchgeführt. Die Fragmentbewegung wurde mit einem optoelektronischen 3-D-Messsystem aufgezeichnet. Bei allen Modellen waren sämtliche Bohrungen für die Osteosynthese identisch und vor der 1 . Messung eingebracht. Randomisiert wurde mit der herkömmlichen oder der neuen Plattenosteosynthese begonnen. Gemessen wurde sowohl die Druck- und Kolbenstrecke als auch die absolute und relative Bewegung des Beckenmodells.

Ergebnisse: Beide Gruppen waren hinsichtlich der Frakturbewegung und der Fragmentverkippung sehr ähnlich. Die neue Flügelplatte konnte insgesamt eine etwas höhere Stabilität aufweisen als die Versuchsreihe mit der herkömmlichen Plattenosteosynthese. Bei der herkömmlichen Platte kam es in 2 Fällen zu einem Plattenausbruch. Beide Modelle konnten für die Versuchsdurchführung mit der Flügelplatte weiterverwendet und voll belastet werden. Aufgrund der guten

\section{Abstract \\ $\nabla$}

Background: Acetabular fractures are rare injuries, but there is an increasing number of elderly people with ventral medial instability in cases of central subluxation of the femoral head in osteoporotic acetabular fractures. Common plate osteosynthesis cannot enable medial support of the quadrilateral surface. The new "acetabular wing plate" is anatomically shaped to fix the arcuate line and the quadrilateral surface. The plate pushes the femoral head back to lateral. The aim of this study was the biomechanical comparison with common plate concepts before clinical use of the new implant.

Methods: For biomechanical testing eight artificial fracture models of the pelvis with anterior column fractures were used. They were implemented into a set-up of a one-leg stand model in the material test machine. Cyclical movements with axial pressure to the sacrum up to $250 \mathrm{~N}$ were given to the model. Fracture gap movement was measured with an optoelectronic 3D camera measuring system. In all pelvic models all screw holes were drilled by use of drill guides before first mechanical loading. The measurements were randomly done first with the new acetabular wing plate or with the pelvic low profile plate. Absolute and relative fracture gap movement and movements of the pelvic fragments were measured as well.

Results: The fracture gap movement and the fragment rotation were comparable in both groups. There was a slightly higher stability in the group of the acetabular wing plate but without statistical significance. In the pelvic low profile group two screw loosenings were found, but the models were able to be tested also with the acetabular wing plate. The new plate was clinically used in the first eight patients due to the good biomechanical results. In all cases the osteosyntheses were done by use of the Stoppa approach and the 
biomechanischen Ergebnisse erfolgte bereits der erste klinische Einsatz. In 8 Fällen wurde die Platte verwendet, jeweils über einen Stoppa-Zugang kombiniert mit dem 1. Fenster des ilioinguinalen Zugangs. Die Applikation der Platte gestaltete sich dabei zumeist einfach, sie konnte sogar als Repositionshilfe verwendet werden. Die postoperativen Kontrollen zeigten eine regelrechte Reposition und korrekte Plattenplatzierung. Das 3-Monats-Follow-up ergab keinen sekundären Repositionsverlust. first window of the ilioinguinal approach. Application of the plate was mostly easy, the plate was also used as a reduction tool. Postoperative controls show anatomic reduction and correct implant position in all eight cases. The three month follow-up examinations confirm the continuing good reduction during fracture healing with the acetabular wing plate.

\section{Einleitung}

Azetabulumfrakturen sind selten [1], können aber bei der Versorgung eine Herausforderung für den Operateur darstellen [1,2]. Typischerweise resultieren Azetabulumfrakturen als Folge eines Hochrasanztraumas. Hierbei kommt es häufig zu einer Verletzung der hinteren Wand und/oder des hinteren Pfeilers [3], gefolgt von T-Frakturen [4]. In den letzten Jahren deutlich zunehmend sind jedoch Azetabulumfrakturen infolge von Niedrigenergietraumata bei älteren Patienten mit osteoporotischer Knochenstruktur. Hier entsteht die Fraktur durch die Protrusion des Hüftkopfs. Die Frakturen weisen neben einer ventralen Hauptkomponente zumeist auch eine Medialisierung der quadrilateralen Fläche, z.T. mit Imprimat des Pfannendachfragments (Gull Sign) [5-7], auf. Typische Frakturformen sind hierbei die Fraktur des vorderen Pfeilers mit hinterer Hemiquerfraktur sowie die Zweipfeilerfraktur [8].

Bei multifragmentären osteoporotischen Frakturen ist es oft schwierig, durch die flächige Abstützung mit den bewährten schmalen Plattensystemen den sekundären Korrekturverlust zu verhindern, weshalb in der Vergangenheit von einigen Autoren auch eine aufwendige Implantation einer Hüfttotalendoprothese mit Abstützring oder sogar die konservative Behandlung erwogen wurde [5,9]. Jedoch sind die Standzeiten von Hüftendoprothesen direkt nach Azetabulumfrakturen kürzer als nach primären Hüftendoprothesen $[10,11]$ und Revisionsoperationen nach schon primär eingebrachten Abstützringen aufwendiger [11]. Vor dem Hintergrund steigender Zahlen osteoporotischer Azetabulumfrakturen bei Menschen mit einem hohen körperlichen Aktivitätsanspruch steigt auch das Bestreben, durch eine anatomische Osteosynthese nach Azetabulumfraktur einen festen Knochenstock zu rekonstruieren, der ein gutes funktionelles Ergebnis ermöglicht oder bei Ausbildung einer posttraumatischen Arthrose einen begrenzt invasiven Oberflächenersatz an der Hüfte gestattet. Die funktionellen Ergebnisse konservativ behandelter, zentral luxierter Azetabulumfrakturen sind nicht zufriedenstellend und häufig mit sekundären Komplikationen behaftet [11].

Aus dieser Situation heraus entstand der Anspruch, ein Implantat zu entwickeln, das eine flächige Reposition und Abstützung zentral dislozierter, multifragmentärer Azetabulumfrakturen gestattet und über wenig invasive Zugangswege auch bei älteren Patienten eine anatomische Rekonstruktion ermöglichen soll.

Die klassische Osteosynthese erfolgt über einen ilioinguinalen Zugang mit Fixation einer langen Platte entlang der Beckeneingangsebene ( $\bullet$ Abb. 1) [12]. Die Stabilität wird über lange Schrauben vom vorderen in den hinteren Pfeiler entlang der quadrilateralen Fläche erreicht. Diese Schrauben können dabei eine Länge von 80 bis $90 \mathrm{~mm}$ haben, teilweise sogar über $100 \mathrm{~mm}$.

In den letzten Jahren hat sich bei diesen zentralen, mehrfragmentären Frakturkomponenten auch der modifizierte StoppaZugang bewährt. Hierüber kann auch eine vorkonturierte Platte von innen angebracht werden [13], die der Medialisierung entgegenwirkt ( $\bullet$ Abb. 1). Die zumeist eingebrachte Kleinfragmentplatte hat jedoch nur eine geringe Auflagefläche und kann insbesondere bei mehrfragmentären Frakturen nur ein teilweise ungenügendes Widerlager bieten [5].

Die Anforderungen an die Entwicklung einer neuen Platte bestehen in:

- vergleichbarer Stabilität der Osteosynthese gegenüber aktuellen Verfahren,

- flächiger Abstützung der quadrilateralen Fläche,

- einfacher Applikation und

- der Möglichkeit zusätzlicher Schraubeneinbringung über das 1. Fenster zur Fixierung des hinteren Pfeilers gegen den vorderen Pfeiler.

Als Resultat wurde eine „Azetabulum-Flügelplatte (acetabula wing plate)“ entwickelt ( $\bullet$ Abb. 2).

\section{Material und Methoden}

$\nabla$

Verwendet wurden 8 handelsübliche Kunststoffmodelle der Firma Synbone ${ }^{\circledR}$ (Malans, Schweiz) mit bereits eingebrachter Fraktur des vorderen Pfeilers, hoch auslaufend, und Beteiligung der quadrilateralen Fläche [14]. Nachempfunden wurde der Einbeinstand. Hierfür wurde ein Beckenmodell in einer Prüfmaschine justiert, sodass eine zentrale Krafteinleitung über das Os sacrum und die rechte „Hüfte“ möglich war. Als Widerlager wurde eine an der Bodenplatte fixierte Duokopfprothese verwendet [15].

Für die kontrollierte Krafteinleitung wurde das Beckenmodell auf der frakturierten Seite auf einer Duokopfprothese gelagert und die Belastung über einen Sockel aus Hartschaum im Sinne eines LWK-V-Ersatzes in das Os sacrum eingeleitet. Durch eine Simulation des dorsalen Bandapparats mit standardisiert gespannten Kabelbindern wurde die Kraft auf die belastete Beckenschaufel übertragen. Analog wurde die Symphyse durch standardisiert eingebrachte Bänder verstärkt. Zur Stabilisierung des Beckenmodells in der Horizontalen bei Krafteinleitung über die Wirbelsäule diente eine ebenfalls standardisiert eingebrachte Simulation der Glutealmuskulatur, die durch vorgedehnte Seile mit einer Reißkraft von über 500Nm den Zug von der Beckenschaufel auf einen dem Trochanter major entsprechenden Ansatzpunkt überträgt.

Durch eine in der sagittalen Ebene variable Winkeleinstellung der Krafteinleitung in LWK V und durch die Verwendung einer in a.-p. Richtung angeordneten Walze zur Krafteinleitung in die Deckplatte von LWK V konnte auf zusätzliche abstützende oder balancierende Maßnahmen verzichtet werden ( Abb.3) [16, 17], wie sie durch andere Autoren für Beckeneinbeinstandmodelle in der Literatur angegeben worden sind [17], dabei jedoch kein natürliches Pendant im Menschen haben.

Vor Beginn der Messzyklen wurde bei jedem Beckenmodell der Winkel in LWK V im Scharnier über 2 Schrauben feinjustiert, so- 


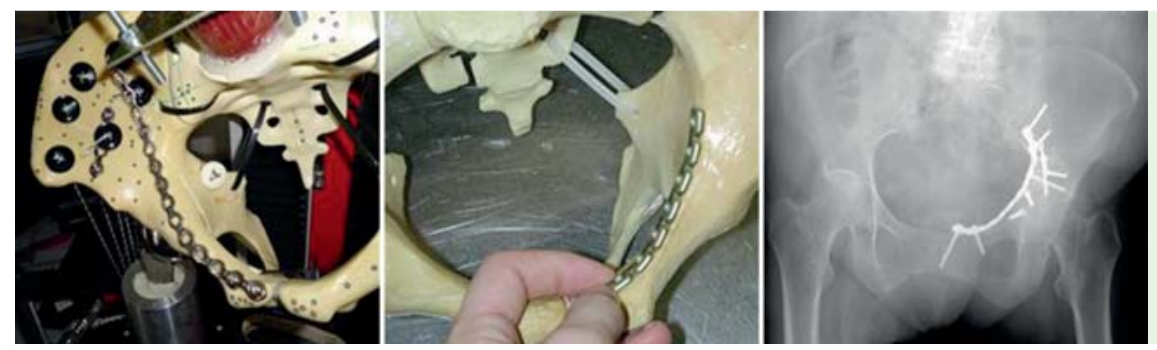

Abb. 1 Beckenmodell mit typischer Lage der 3,5mm-J-Platte links, Position der von medial eingebrachten Platte am Modell; Beispiel einer 86-jährigen Patientin, mit typischer Osteosynthese über einen Stoppa-Zugang versorgt.
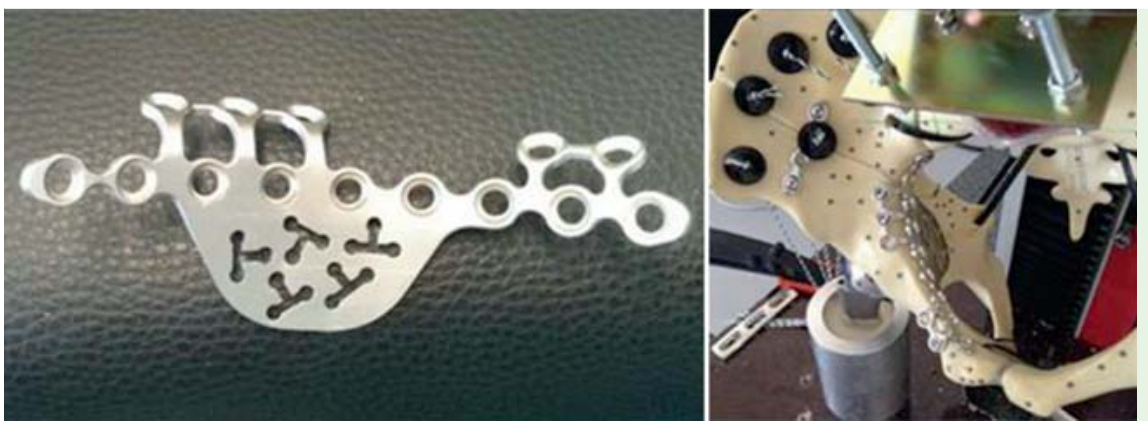

Abb. 2 Die neue Azetabulum-Flügelplatte links, Beckenmodell mit einliegender „Flügelplatte“ rechts.

Tab. 1 Randomisierte Reihenfolge der Versuchsdurchführung.

\begin{tabular}{|lllllllll} 
Platte & \multicolumn{2}{l}{ Position } & & & & & & \\
Becken LP & 2 & 1 & 2 & 1 & 1 & 2 & 2 & 1 \\
\hline Flügel & 1 & 2 & 1 & 2 & 2 & 1 & 1 & 2
\end{tabular}

dass bei axialer Belastung von oben das Becken in der Sagittalebene nach links ausweichen und nach der Kraftreduktion selbstständig wieder in die Ausgangslage zurückgleiten konnte (๑ Abb. 3).

Mit einer Materialprüfmaschine (Zwick GmbH \& Co. KG, Ulm, Modell zwicki-Line ${ }^{\circledR}$ ) wurde eine trizyklische Messung mit jeweils steigenden Belastungsstufen von 50, 100, 150, 200 und $250 \mathrm{~N}$ durchgeführt. Zwischen den einzelnen Messzyklen wurde das System wieder in die Ausgangsposition zurückgefahren und überprüft, ob es zu einer plastischen Verformung gekommen war. Da es im Rahmen eines Vorversuchs bei 320 bzw. 330 N zu einem Schraubenausbruch gekommen war ( Abb. 13), wurde für die Messreihe die Belastung bis $250 \mathrm{~N}$ gemessen (s.u.). Die Reihenfolge der Versuchsdurchführung der Osteosyntheseverfahren erfolgte randomisiert ( $\bullet$ Tab. 1 ).

Im Messbereich des optischen Messsystems wurden multiple optische Messpunkte über das gesamte Beckenmodell verteilt. Die Marker wurden mit einem optischen Messsystem (PONTOS ${ }^{\circledR}$ der Firma $\mathrm{GOM}^{\circledR}{ }^{\circledR}$, Braunschweig) erfasst und deren Bewegung 3-dimensional festgehalten ( $\triangle$ Abb. 10) [18]. Die Auswertung erfolgte im Anschluss anhand der Positionsveränderung der Marker zueinander und im Raum. Für die Auswertung wurden die 3 Fragmente des Modells als „hinterer Pfeiler“, „,vorderer Pfeiler“ und „quadrilaterale Fläche“ bezeichnet.

Als Osteosynthese wurde die von uns in Zusammenarbeit mit der Firma DePuy Synthes ${ }^{\circledR}$ (Zuchwil, Schweiz) entwickelte Flügelplat-

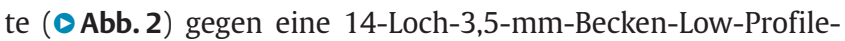
Platte (LP, Stahl; DePuy Synthes) als Standardosteosynthese ( Abb. 1) verglichen, wobei bei beiden Osteosynthesen der hohe Anteil des vorderen Pfeilers zusätzlich mit einer 4-Loch-Stahl-LPPlatte gegen den hinteren Pfeiler stabilisiert wurde ( $\bullet$ Abb. 4).

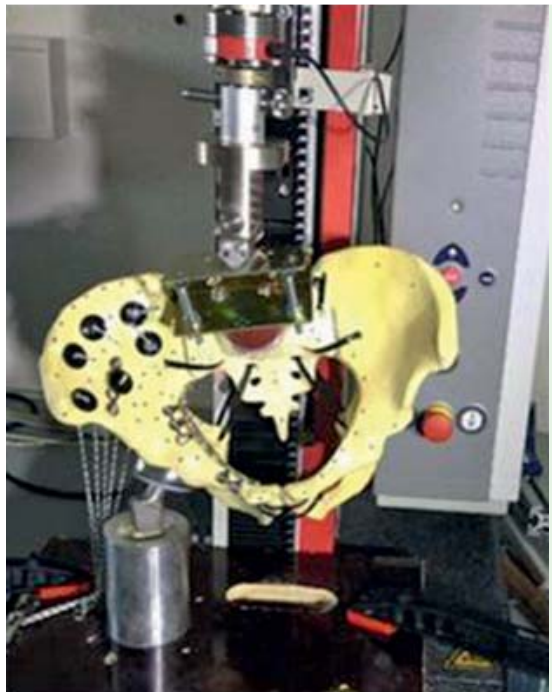

Abb. 3 Beckenmodell bereit für Messzyklus mit der neuen Flügelplatte.

\section{Ergebnisse \\ $\nabla$}

Ausgewertet wurde zum einen die Abstandsänderung an 2 Stellen der Fraktur ( $\bullet$ Abb.5), zum anderen die Rotation der einzelnen Fragmente bez. ihrer Raumposition ( $\triangle$ Abb. 8). Unterschieden wurde hier der Nick-, Gier- und Rollwinkel. Der Nickwinkel entspricht einer Rotation in der Frontalebene, der Gierwinkel in der Axialebene und der Rollwinkel in der Sagittalebene ( $\bullet$ Abb. 9). Beide Messreihen waren hinsichtlich der Frakturbewegung und der Fragmentverkippung insgesamt sehr ähnlich. Bei der Versuchsreihe mit der Flügelplatte konnte eine tendenziell höhere Stabilität ohne statistische Signifikanz gefunden werden. Nach statistischer Analyse der gewonnenen Daten über einen t-Test zeigte sich, dass eine Erhöhung der Anzahl untersuchter Beckenmodelle bis zur Erzielung signifikanter Ergebnisse bei einer Power von 90\% mit dem vorliegenden Studienbudget nicht umsetzbar war. 

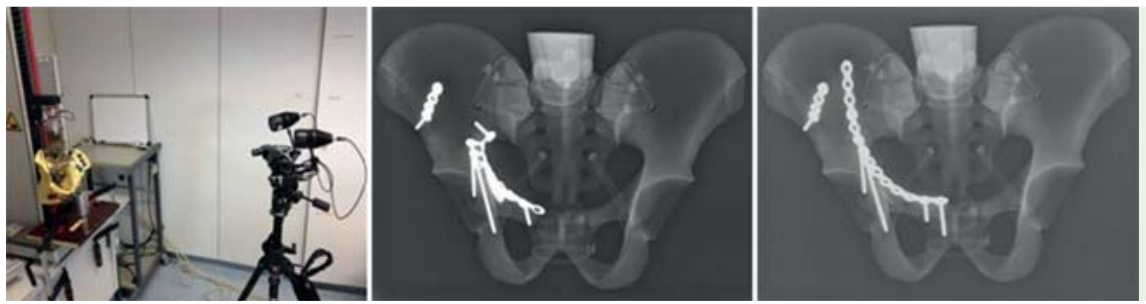

Abb. 4 Versuchsanordnung: Bild links: die Prüfmaschine mit eingespanntem Beckenmodell und optischem Messsystem; Bild in der Mitte: Röntgenbild des Beckenmodells mit angebrachter Flügelplatte; Bild rechts: Röntgenbild des Beckenmodells mit angebrachter J-Platte.

In allen Fällen konnte unter Belastung ein sehr gutes Repositionsergebnis gehalten werden.

\section{Die Auswertung im Einzelnen}

Abstandmessung

Bei der Auswertung der Frakturspaltbewegung fanden sich geringe Unterschiede in beiden Gruppen. Die plattenferne Fragmentbewegung bei der neuen Flügelplatte war an der lateralen Messstelle bei 7 von 8 Fällen geringer als bei der herkömmlichen Plattenosteosynthese ( Abb.6). Im Durchschnitt wurde eine Frakturspaltbewegung bei der „alten Platte“ von $0,25 \mathrm{~mm}$ in XRichtung, 0,53 $\mathrm{mm}$ in Y-Richtung und $0,55 \mathrm{~mm}$ in Z-Richtung, bei der neuen Flügelplatte von $0,35 \mathrm{~mm}$ in X-Richtung, 0,54 mm in Y-Richtung und 0,54 $\mathrm{mm}$ in Z-Richtung gemessen ( $\bullet$ Abb. 7).

\section{Verkippung der Fragmente}

Die Flügelplatte hat bei 7 von 8 Messzyklen eine geringere Verkippung der Fragmente „vorderer Pfeiler“ zu „hinterer Pfeiler“ ( Abb. 11) im Nickwinkel als die herkömmliche Platte gezeigt. Mithilfe der Azetabulum-Flügelplatte konnte das quadrilaterale Fragment besser stabilisiert werden. Durchschnittlich wurde die Verkippung der quadrilateralen Fläche zum vorderen Pfeiler von $0,97^{\circ}$ mit der herkömmlichen Platte auf $0,72^{\circ}$ mit der Flügelplatte reduziert. Die Verkippung bez. des hinteren Pfeilers konnte von $1,08^{\circ}$ auf $0,78^{\circ}$ verbessert werden. Die Winkel zwischen dem vorderen und dem hinteren Pfeilerfragment waren mit $0,42^{\circ}$ bei der Flügelplatte und $0,47^{\circ}$ bei der J-Platte sehr ähnlich.

Bei der Messmethode mit der herkömmlichen Platte kam es im Vorversuch 2-mal (320 N und $330 \mathrm{~N}$ ) zu einem Plattenausbruch

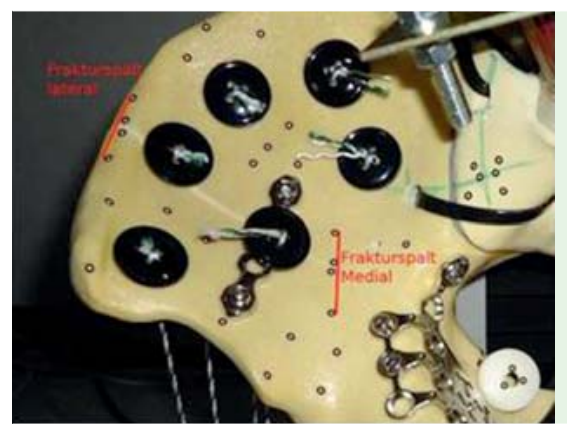

Abb. 5 Definition der Messpunkte für die Abstandsmessung.

( Abb. 12). Beide Modelle konnten für die Versuchsdurchführung mit der Flügelplatte weiterverwendet und sogar bis $350 \mathrm{~N}$ belastet werden. Als Konsequenz wurde bei der Versuchsreihe auf eine Belastung über $250 \mathrm{~N}$ verzichtet.

\section{Diskussion}

$\nabla$

Die Applikation der neuen, vorkonturierten Platte war in allen Fällen einfach möglich mit hoher Passgenauigkeit zum Beckenmodell. Die biomechanische Versuchsreihe hat gezeigt, dass die neu entwickelte anatomische Azetabulum-Flügelplatte vergleichbar gute mechanische Ergebnisse wie die bewährte Plattenosteosynthese liefert. Tendenziell fand sich bei der Flügelplatte eine höhere Stabilität, jedoch ohne statistische Signifikanz. Die Frakturspaltbewegungen waren für beide Osteosyntheseverfah-

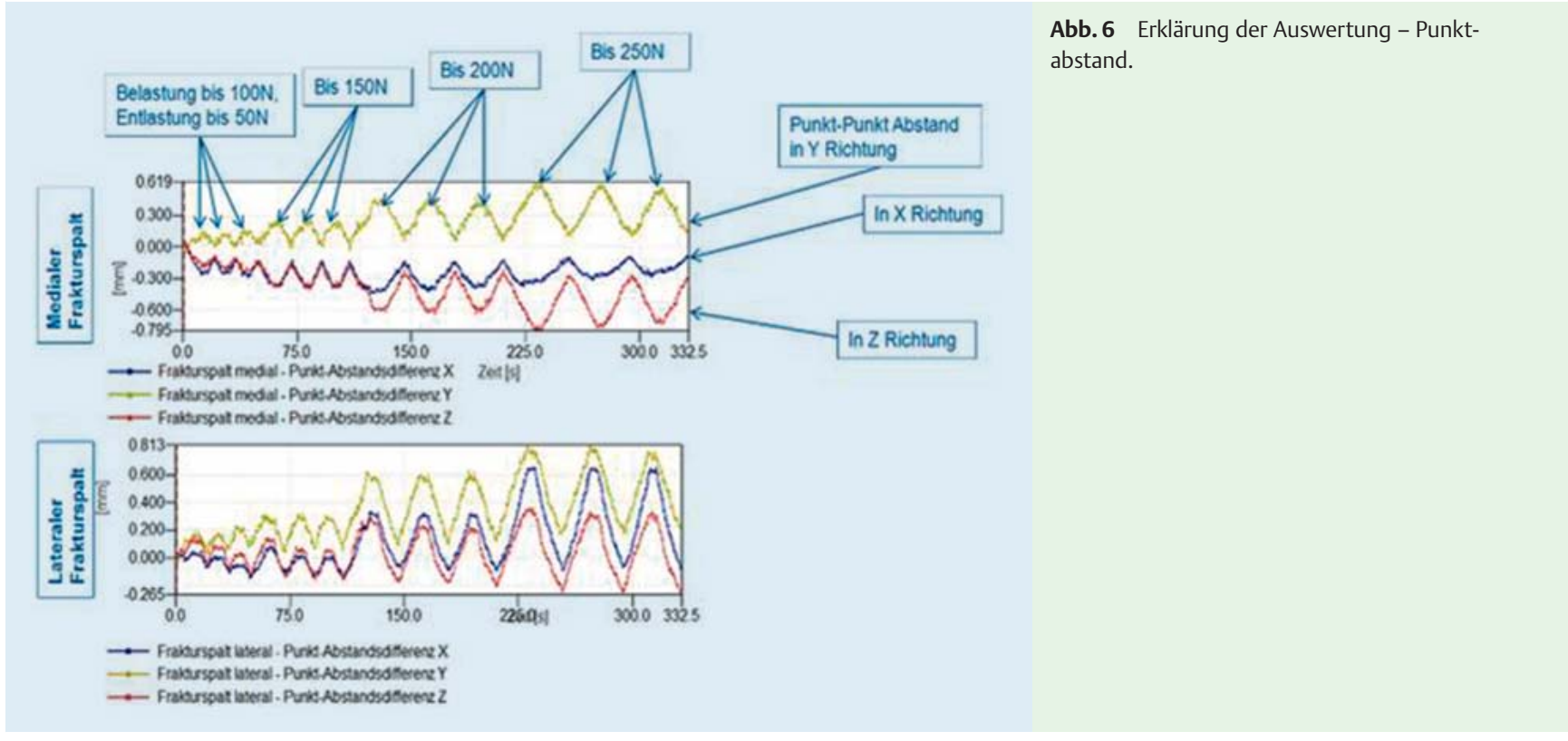




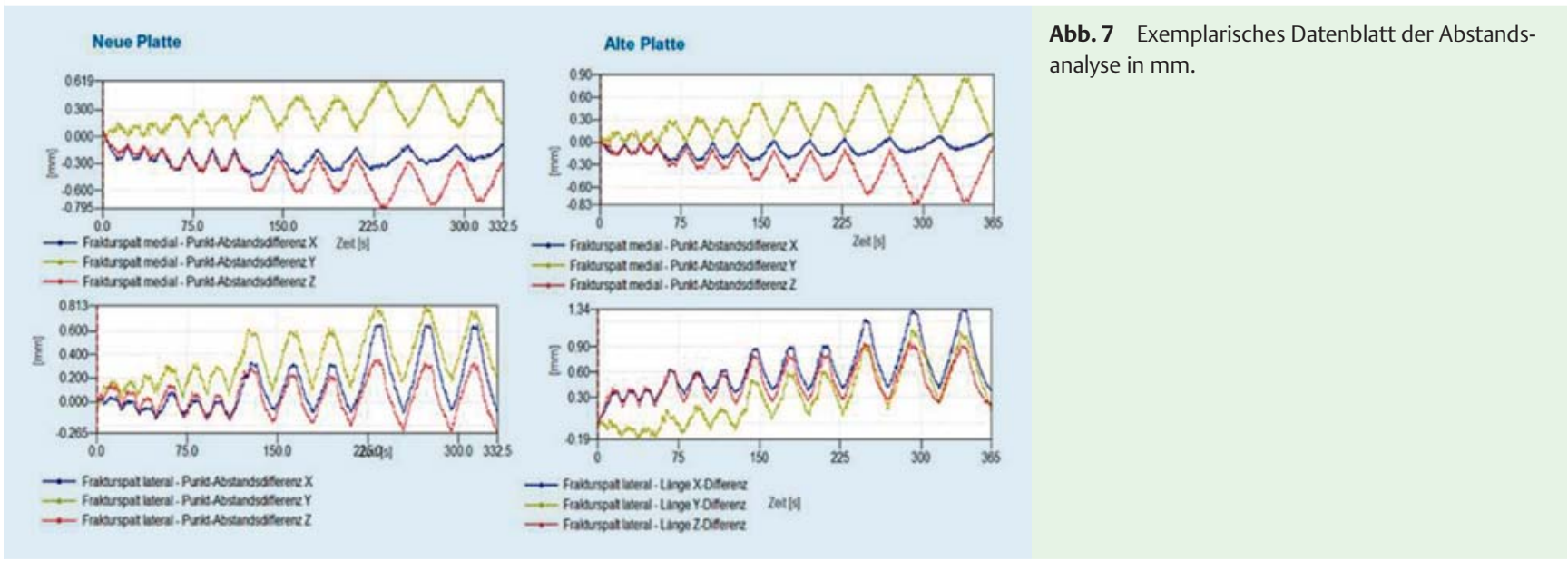

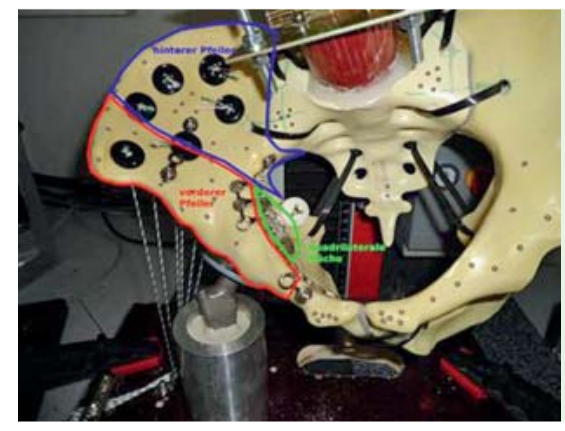

Abb. 8 Darstellung der einzelnen Ebenen für die Messung der Fragmentverkippung. Rot: „vorderer Pfeiler“; blau: „hinterer Pfeiler“; grün: „quadrilaterale Fläche“. ren vergleichbar gering. Die Auswertung der Fragmentverkippung zeigte mit der Flügelplatte eine konstant geringere Torquierung zwischen dem vorderen und hinteren Pfeilerfragment. Besonders eindrücklich war die veränderte Stabilität bei der Fixierung der quadrilateralen Fläche. Zu berücksichtigen ist dabei, dass die Versuche an einem normalen Knochenmodell durchgeführt wurden. Bei einem osteoporotischen Knochenmodell wären wohl eher deutlichere Unterschiede zu erwarten.

Die axiale Krafteinleitung bis $250 \mathrm{~N}$ über das Os sacrum spiegelt nicht die Hüftanpresskräfte im Azetabulum wider, die in dem verfügbaren Setup nicht gemessen werden konnten. Andere Autoren nehmen bei vergleichbarem Setup die Belastung im Einbeinstand [21] mit $800 \mathrm{~N}$ entsprechend einer Vollbelastung an [14]. Im hier verwendeten Setup konnte nach Vorversuchen wegen bauartbedingt zunehmender Dislokation der SI-Fugen eine solche Belastung nicht durchgeführt werden.

Die Fixierung der quadrilateralen Fläche wird häufig als schwierig beschrieben und von einigen Autoren, neben der Verletzung des Domfragments, als einer der entscheidenden prognostischen Faktoren für das Langzeitergebnis gesehen. In einer CochraneAnalyse in der Zeitschrift Injury 2013 wurden alle englischsprachigen Publikationen, die sich mit der Problematik dieser speziellen anatomischen Region beschäftigen, analysiert und ausgewertet [5]. Verglichen wurden die konservative Therapie gegen die operative sowie die einzelnen OP-Methoden. Insgesamt wurden 1573 Studien beurteilt. Daraus konnten 16 inkludiert werden. Die Studie analysierte Veröffentlichungen zwischen 1956 und 2012.

Als Quintessenz der Studie sahen die Autoren, dass bis in die 60er-Jahre hauptsächlich eine konservative Behandlung propagiert wurde, die jedoch einer langen Zeit der Teilbelastung
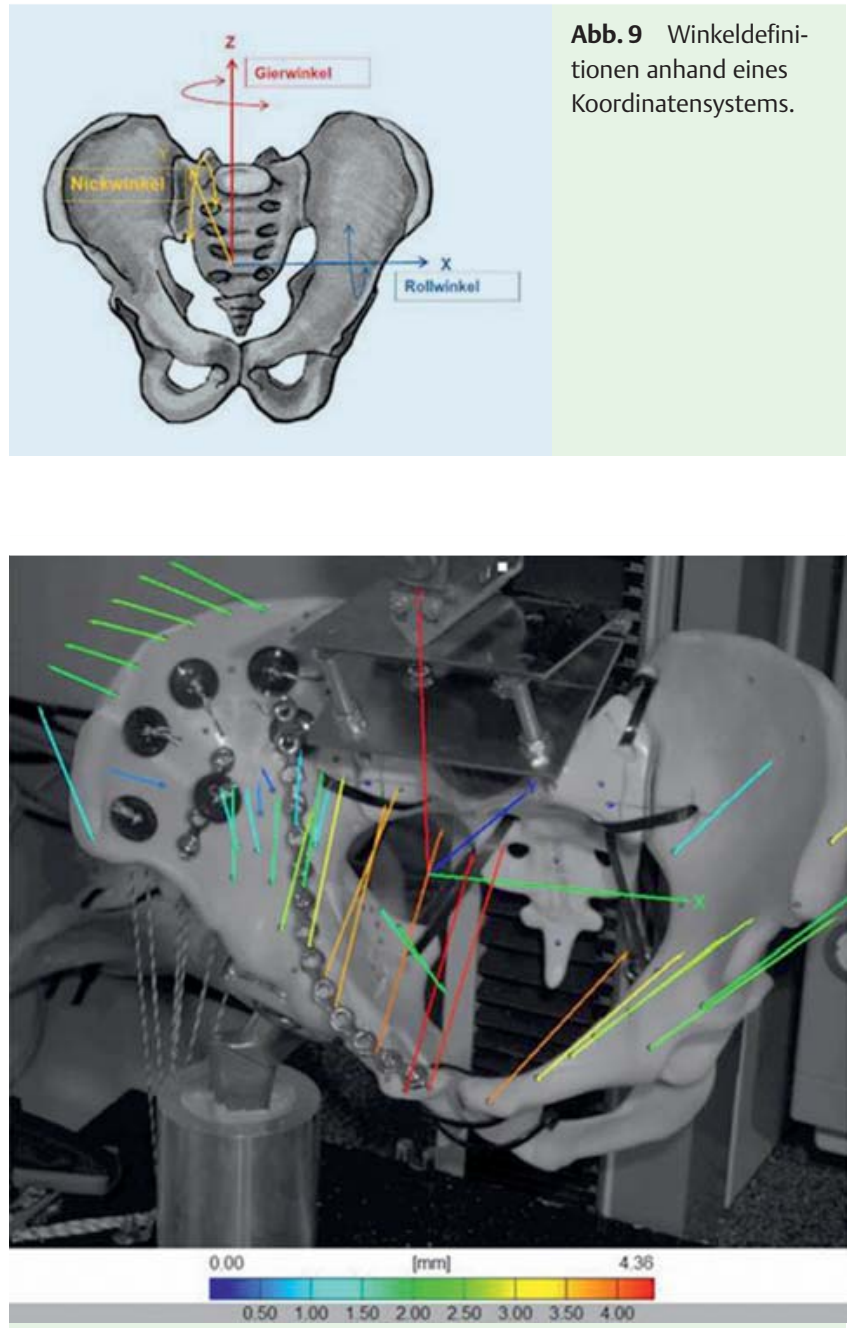

Abb. 10 Farbige Darstellung der Relativbewegung in $\mathrm{mm}$.

und teilweise auch Bettruhe mit angelegtem Extensionsgestell bedurfte [5]. Da die Verletzung der quadrilateralen Fläche jedoch vermehrt bei älteren Patienten auftritt, ist diese Therapie heute nicht mehr anzustreben. Die Risiken der Begleitproblematik wie Thrombosen, Herz-Kreislauf-Versagen oder Pneumonie sind zu groß $[22,23]$. 


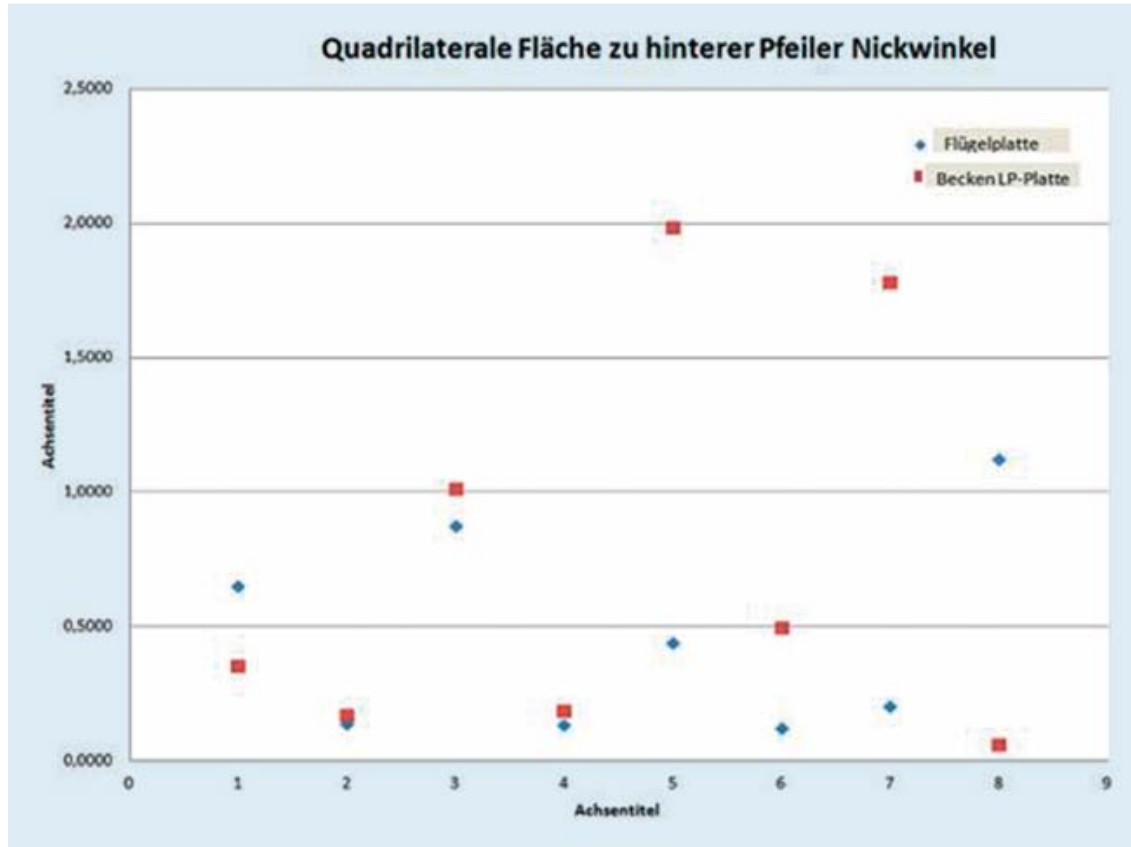

Abb. 11 Exemplarisches Datenblatt der Winkelanalyse.
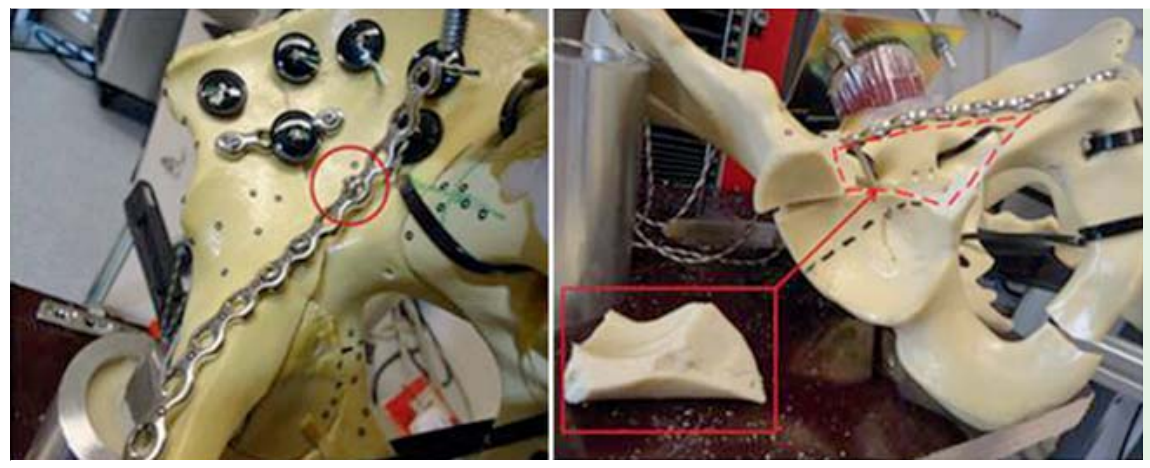

Abb. 12 Plattenausbruch in einer Vorversuchsreihe, beide bei der herkömmlichen Plattenosteosynthese: links: Schraubendislokation bei $330 \mathrm{~N}$, rechts: Bruch im Bereich des vorderen Pfeilers bei $320 \mathrm{~N}$.
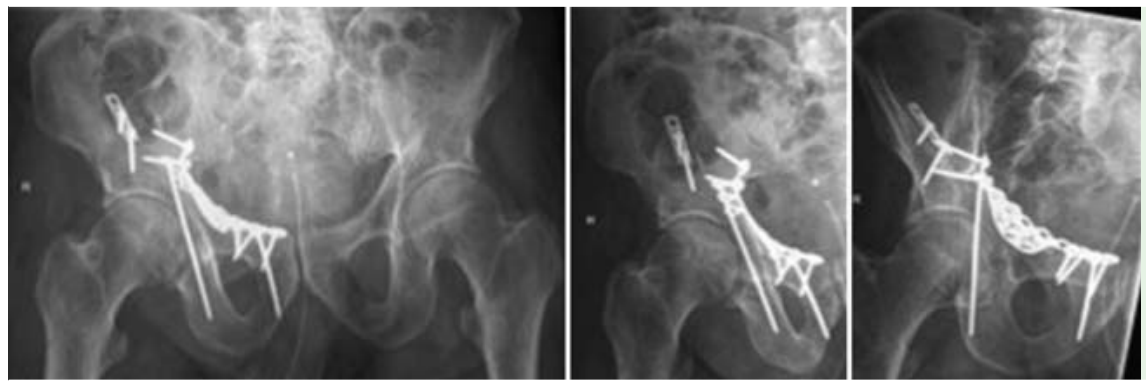

Abb. 13 Postoperative Verlaufskontrolle des 86-jährigen Patienten 6 Tage nach operativer Versorgung mit der neuen Azetabulum-Flügelplatte (Beckenübersicht, Ala-, Obturator-Aufnahme).

In der jüngeren Literatur wird laut der Analyse vermehrt die operative Therapie angeraten, jedoch wird die Fixierung der quadrilateralen Fläche oftmals als großes Problem beschrieben. Einige Autoren betrachten die unzureichende Reposition als Problem der richtigen Zugangswahl, für andere Autoren liegt das Problem in der stabilen Abstützung dieser anatomischen Struktur [5]. Laflamme et al. [24] konnten über einen modifizierten Stoppa-Zugang in 52,4\% eine anatomische Reposition mit sehr guten Ergebnissen zeigen. Jedoch kam es bei 4 Fällen zu einem sekundären Korrekturverlust und dadurch zu einem raschen Fortschreiten der Arthrose. Chen et al. 2001 [25] und Farid 2010 [26] beschreiben die zusätzliche Absicherung mit einer Draht- bzw. Kabel-Cer- clage zur besseren Abstützung. Die Autoren sehen die Einbringung als weitgehend sicher und unkompliziert an. Aktuell am weitesten verbreitet dürfte wohl die Abstützung mittels sog. Spring Plate sein. Tscherne und Pohlemann empfehlen in ihrem Buch über die Beckenchirurgie zur besseren Abstützung hierfür bereits 1998 die Verwendung der breiteren H-Platte. Unabhängig von anderen OP-Methoden wird durch den Einsatz der 3-D-Bildwandler im OP das Repositionsergebnis dank der intraoperativen CT-ähnlichen Kontrolle deutlich verbessert [27]. Erkenntnisse über eine tatsächliche Verbesserung der Langzeitergebnisse nach einer Azetabulumverletzung liegen jedoch in der Literatur noch nicht vor. 


\section{Medizinischer Einsatz und Ausblick}

$\nabla$

Die Testreihe erbrachte eine zumindest zur aktuellen Plattenosteosynthese vergleichbare stabile Osteosynthese. Die neue Flügelplatte stabilisiert hierbei gleichzeitig die quadrilaterale Fläche. Die Applikation erfolgt weichteilschonend über den modifizierten Stoppa-Zugang alleine oder in Kombination mit dem 1. Fenster nach Letournel [28-30], denkbar wäre auch die Verwendung des Pararectus-Zugangs [31], wie er von Keel et al. beschrieben wurde. Damit konnten die ursprünglichen Ziele der Entwicklung erfüllt werden.

Wir haben bei 8 Patienten diese Platte bereits angewandt. Die Platzierung war über den Stoppa-Zugang in Kombination mit dem 1. Fenster in allen Fällen sicher möglich. Wir konnten in allen Fällen ein patientenspezifisches Implantat nach dem Vorbild der Flügelplatte gestalten und darüber eine gute Reposition erreichen ( Abb. 13). Im 3-Monats-Follow-up konnte kein sekundärer Repositionsverlust beobachtet werden. Eine prospektive Studie mit größerer Fallzahl ist notwendig, um die klassische Anwendbarkeit besser zu beurteilen.

\section{Fazit}

$\nabla$

Der erste klinische Einsatz der patientenspezifischen anatomischen Azetabulum-Flügelplatte konnte neben der biomechanischen Stabilität das gute Handling zur Reposition mehrfragmentärer Frakturen der quadrilateralen Fläche mit zentraler Dislokation bestätigen, auch wenn das biomechanische Setup nicht die klinische Belastungssituation bei alten Menschen mit oft fehlender Fähigkeit zur Teilbelastung wiedergeben kann. Für die Serienreife dieser Platte müssen jedoch noch kleinere Anpassungen vorgenommen werden, um den variablen anatomischen Gegebenheiten gerecht zu werden.

Wir sehen bei der Flügelplatte das vordringliche Einsatzgebiet bei Frakturen mit zentraler Luxation vor allem bei älteren osteoporotischen Menschen, da hier die herkömmlichen Verfahren keine ausreichende Abstützung der quadrilateralen Fläche zugelassen haben. Bei steigender Patientenzahl mit osteoporotischen Azetabulumfrakturen und hohem körperlichen Anspruch steigt der Bedarf an ein solches Implantat.

\section{Interessenkonflikt: Nein}

\section{Literatur}

1 Pohlemann T, Tscherne H, Baumgartel F et al. [Pelvic fractures: epidemiology, therapy and long-term outcome. Overview of the multicenter study of the Pelvis Study Group]. Unfallchirurg 1996; 99: 160-167

2 D'Imporzano M, Liuni FM, Tarantino U. Acetabular fragility fractures in elderly patients. Aging Clin Exp Res 2011; 23: 71-73

3 Tscherne H, Regel G, Pape HC et al. Internal fixation of multiple fractures in patients with polytrauma. Clin Orthop Relat Res 1998, 347: 62-78

4 Blum J, Beyermann K, Ritter G. [Incidence of acetabular fractures before and after introduction of compulsory seatbelt fastening]. Unfallchirurgie 1991; 17: 274-279

5 White G, Kanakaris NK, Faour O et al. Quadrilateral plate fractures of the acetabulum: An update. Injury 2013; 44: 159-167

6 Rommens PM, Wagner D, Hofmann A. [Osteoporotic fractures of the pelvic ring]. Z Orthop Unfall 2012; 150: e107-e118

7 Rommens PM, Wagner D, Hofmann A. Surgical management of osteoporotic pelvic fractures: a new challenge. Eur J Trauma Emer Surg 2012; 38: 499-509
8 Pohlemann T, Stengel D, Tosounidis G et al. Survival trends and predictors of mortality in severe pelvic trauma: estimates from the German Pelvic Trauma Registry Initiative. Injury 2011; 42: 997-1002

9 Amanatullah DF, Cheung Y, Di Cesare PE. Hip resurfacing arthroplasty: a review of the evidence for surgical technique, outcome, and complications. Orthop Clin North Am 2010; 41: 263-272

10 Bellabarba C, Berger RA, Bentley CD et al. Cementless acetabular reconstruction after acetabular fracture. J Bone Joint Surg Am 2001; 83: 868-876

11 Mears DC, Velyvis JH. Acute total hip arthroplasty for selected displaced acetabular fractures: two to twelve-year results. J Bone Joint Surg Am 2002; 84: 1-9

12 Siebenrock KA, Tannast M, Bastian JD et al. [Posterior approaches to the acetabulum]. Unfallchirurg 2013; 116: 221-226

13 Qureshi AA, Archdeacon MT, Jenkins MA et al. Infrapectineal plating for acetabular fractures: a technical adjunct to internal fixation. J Orthop Trauma 2004; 18: 175-178

14 Marintschev I, Gras F, Schwarz CE et al. Biomechanical comparison of different acetabular plate systems and constructs-the role of an infraacetabular screw placement and use of locking plates. Injury 2012; 43: 470-474

15 Tabaie SA, Bledsoe JG, Moed BR. Biomechanical comparison of standard iliosacral screw fixation to transsacral locked screw fixation in a type $C$ zone II pelvic fracture model. J Orthop Trauma 2013; 27: 521-526

16 Pohlemann T, Angst M, Schneider E et al. Fixation of transforaminal sacrum fractures: a biomechanical study. J Orthop Trauma 1993; 7: 107117

17 Culemann U, Pohlemann T, Hufner T et al. [3-dimensional movement analysis after internal fixation of pelvic ring fractures. A computer simulation]. Unfallchirurg 2000; 103: 965-971

18 Doebele S, Siebenlist S, Vester $H$ et al. New method for detection of complex 3D fracture motion verification of an optical motion analysis system for biomechanical studies. BMC Musculoskelet Disord 2012; 13: 33

19 Bergmann G, Deuretzbacher G, Heller $M$ et al. Hip contact forces and gait patterns from routine activities. J Biomech 2001; 34: 859-871

20 Heller MO, Bergmann G, Deuretzbacher G et al. Musculo-skeletal loading conditions at the hip during walking and stair climbing. J Biomech 2001; 34: 883-893

21 Culemann U, Holstein JH, Kohler D et al. Different stabilisation techniques for typical acetabular fractures in the elderly-a biomechanical assessment. Injury 2010; 41: 405-410

22 Toro JB, Hierholzer C, Helfet DL. Acetabular fractures in the elderly. Bull Hosp Jt Dis 2004; 62: 53-57

23 Cornell CN. Management of acetabular fractures in the elderly patient. HSS J 2005; 1 : 25-30

24 Laflamme GY, Hebert-Davies J, Rouleau D et al. Internal fixation of osteopenic acetabular fractures involving the quadrilateral plate. Injury 2011; 42: 1130-1134

25 Chen CM, Chiu FY, Lo WH et al. Cerclage wiring in displaced both-column fractures of the acetabulum. Injury 2001; 32: 391-394

26 Farid YR. Cerclage wire-plate composite for fixation of quadrilateral plate fractures of the acetabulum: a checkrein and pulley technique. J Orthop Trauma 2010; 24: 323-328

27 Stöckle U, König B, Schäffler A et al. [Clinical experience with the Siremobil Iso-C(3D) imaging system in pelvic surgery]. Unfallchirurg 2006; 109: 30-40

28 Andersen RC, O'Toole RV, Nascone JW et al. Modified stoppa approach for acetabular fractures with anterior and posterior column displacement: quantification of radiographic reduction and analysis of interobserver variability. J Orthop Trauma 2010; 24: 271-278

29 Cole JD, Bolhofner BR. Acetabular fracture fixation via a modified Stoppa limited intrapelvic approach. Description of operative technique and preliminary treatment results. Clin Orthop Relat Res 1994, 305: 112123

30 Fan L, Jin YJ, He L et al. [Modified Stoppa approach in treatment of pelvic and acetabular fractures]. Zhongguo Gu Shang 2012; 25: 810-812

31 Keel MJ, Ecker TM, Cullmann JL et al. The Pararectus approach for anterior intrapelvic management of acetabular fractures: an anatomical study and clinical evaluation. J Bone Joint Surg Br 2012; 94: 405-411 